\title{
Immense Magnetic Response of Exciplex Light Emission due to Correlated Spin-Charge Dynamics
}

\author{
Yifei Wang, Kevser Sahin-Tiras, Nicholas J. Harmon, Markus Wohlgenannt, and Michael E. Flatté \\ Department of Physics and Astronomy and Optical Science and Technology Center, \\ University of Iowa, Iowa City, Iowa 52242, USA \\ (Received 8 September 2015; revised manuscript received 8 January 2016; published 5 February 2016)
}

As carriers slowly move through a disordered energy landscape in organic semiconductors, tiny spatial variations in spin dynamics relieve spin blocking at transport bottlenecks or in the electron-hole recombination process that produces light. Large room-temperature magnetic-field effects (MFEs) ensue in the conductivity and luminescence. Sources of variable spin dynamics generate much larger MFEs if their spatial structure is correlated on the nanoscale with the energetic sites governing conductivity or luminescence such as in coevaporated organic blends within which the electron resides on one molecule and the hole on the other (an exciplex). Here, we show that exciplex recombination in blends exhibiting thermally activated delayed fluorescence produces MFEs in excess of $60 \%$ at room temperature. In addition, effects greater than $4000 \%$ can be achieved by tuning the device's current-voltage response curve by device conditioning. Both of these immense MFEs are the largest reported values for their device type at room temperature. Our theory traces this MFE and its unusual temperature dependence to changes in spin mixing between triplet exciplexes and light-emitting singlet exciplexes. In contrast, spin mixing of excitons is energetically suppressed, and thus spin mixing produces comparatively weaker MFEs in materials emitting light from excitons by affecting the precursor pairs. Demonstration of immense MFEs in common organic blends provides a flexible and inexpensive pathway towards magnetic functionality and field sensitivity in current organic devices without patterning the constituent materials on the nanoscale. Magnetic fields increase the power efficiency of unconditioned devices by $30 \%$ at room temperature, also showing that magnetic fields may increase the efficiency of the thermally activated delayed fluorescence process.

\section{INTRODUCTION}

Organic light-emitting diodes (OLEDs) [1] based on organic semiconductors are used extensively in flat-panel displays and other lighting, due to their mechanical flexibility and low-cost fabrication. In most OLEDs electrons and holes are injected into the device and, upon encountering one another, form a loosely bound state (a polaron pair) and, finally, a tightly bound exciton. Because both the electron and hole carry spin-1/2, these bound states can be spin singlets (total spin 0), or triplets (total spin 1), and they usually form in a 1:3 ratio based on spin degeneracy. Most organic semiconductors are fluorescent materials with an internal electroluminescence (EL) quantum efficiency $\eta$ limited to $\eta \approx 25 \%$ because only singlet excitons lead to significant electroluminescence [2]. Recently, triplet-to-singlet up-conversion in thermally activated delayed fluorescence (TADF) blends has increased $\eta$ well above $25 \%[3,4]$. TADF requires the exchange

*michael_flatte@mailaps.org

Published by the American Physical Society under the terms of the Creative Commons Attribution 3.0 License. Further distribution of this work must maintain attribution to the author(s) and the published article's title, journal citation, and DOI. splitting between the singlet and triplet states $\Delta_{\mathrm{ST}}$ to be smaller than or comparable to the thermal energy ( $\approx 25 \mathrm{meV}$ at room temperature). As $\Delta_{\mathrm{ST}}$ in excitons is usually orders of magnitude larger, intermolecular excitations, or so-called exciplexes, which have much smaller $\Delta_{\mathrm{ST}}$, are a common choice to achieve TADF. In parallel to these improvements in OLED emission efficiency, recent investigations have identified large magnetic-field sensitive spin effects on the electroluminescence in organic semiconductors [5-13]. Organic semiconductors also possess an intrinsic magnetoresistive effect, termed organic magnetoresistance (OMAR), which, in magnetic fields $B$ of just a few $\mathrm{mT}$, is $\approx 20 \%$ at room temperature in a large variety of organic semiconductors [6,7], and is often accompanied by magnetoelectroluminescence (MEL) [5,6]. A small $\Delta_{\mathrm{ST}}$ suggests that the spin dynamics of recombination in TADF blends could be dramatically modified by a small magnetic field, producing a very large OMAR effect and improving the efficiency of OLEDs.

Here, we show that an immense ( $>50 \%$ ) magnetic-field effect (MFE) occurs to both the resistance and the electroluminescence in an organic blend already known to possess a large internal quantum efficiency due to TADF. The dramatic enhancement of this magnetic-field effect is due to the properties of the singlet-triplet exchange splitting $\Delta_{\mathrm{ST}}$, 
which also explain the temperature dependence of the magnetic-field effects. The devices are fabricated using typical micron-scale fabrication processes, and do not require any nanoscale structuring of the constituent molecules. Thus, this approach appears broadly applicable to a wide variety of organic devices that incorporate TADF blends without complex fabrication. We experimentally demonstrate in our devices a $30 \%$ increase in the power efficiency of light emission at room temperature due to the application of a magnetic field, compared to the efficiency of the same device at zero field.

A theory that includes the effects of the singlet-triplet level structure associated with TADF is developed and the immense effects due to the interaction of these exciplexes with hyperfine fields and $g$-factor variations are calculated. The effects of hyperfine fields and $g$-factor variations oppose each other, so by the demonstrated increased emission with magnetic field we identify $g$-factor variations (i.e., the $\Delta g$ mechanism $[14,15]$ ) as the dominant mechanism of the effect.
We further demonstrate the ability to "condition" the organic devices to the point where the change in luminescence or resistance exceeds an order of magnitude, with the largest changes in excess of $4000 \%$. Conditioning leads to a decrease in the $\Delta_{\mathrm{ST}}$, which enhances the MFE at constant current, and also an undesirable increase in the device resistance. We thus clarify the connection between magnetic-field effects in current and those in voltage, demonstrating that those effects seen in constant voltage can be amplified through various conditioning procedures, whereas those in constant current are less responsive. The size of these magnetic field effects, $>50 \%$ even in unconditioned devices, is the largest reported to date at room temperature.

\section{THEORY}

In this section, the effects of magnetic fields on spatially separated entities, such as the electrons and holes composing exciplexes, are contrasted with the effects of magnetic
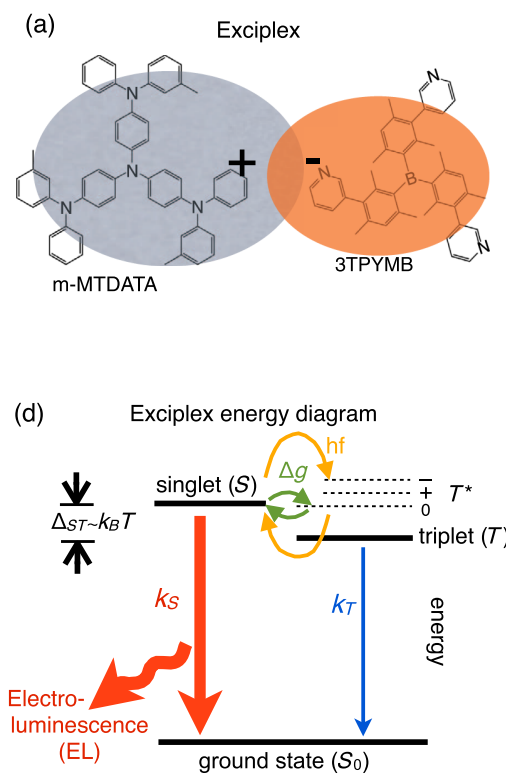

(b) Exciplex spin mixing from $\Delta g$ mechanism

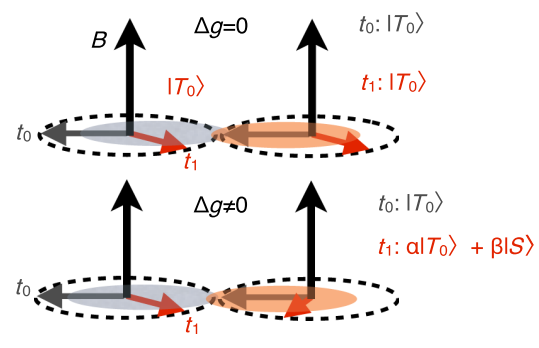

(e)

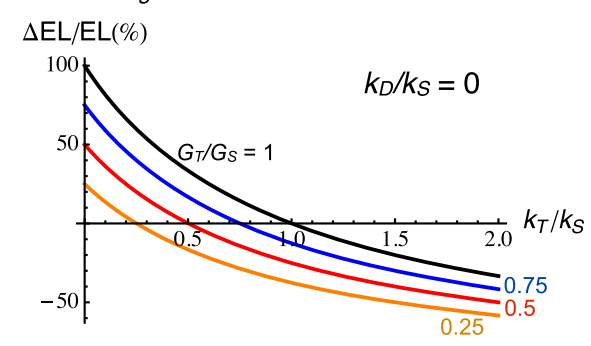

(c)

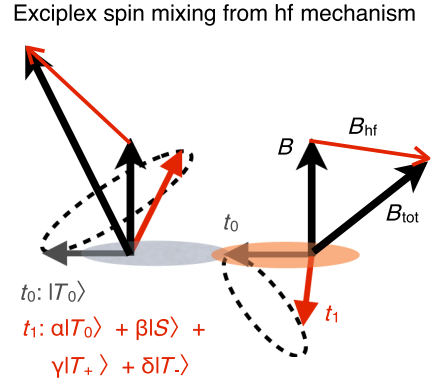

(f)

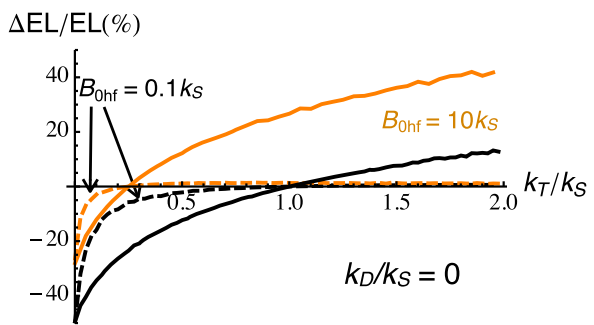

FIG. 1. Exciplexes, $\Delta g$ mechanism, and hyperfine mechanism for magnetic field effects. (a) Schematic of the exciplex with mMTDATA as the donor and 3TPYMB as the acceptor. The electron (hole) wave function is schematically represented by the orange (gray) oval and is overlaid upon the respective chemical structures. (b) $\Delta g$ spin mixing between the electron and hole spins (shown to be orthogonal to the applied field $\boldsymbol{B}$ ) which are initiated at time $t_{0}$ in the $\left|T_{0}\right\rangle$ spin configuration. In the top panel, where there is no difference in $g$ factors, the spins remain in their initial configuration. In the lower panel, with nonzero $\Delta g$, the spinor picks up singlet $(|S\rangle)$ character over time $t_{1}$. (c) As in (b), but hyperfine spin mixing between electron and hole spins. In this case, spin mixing occurs among all four spin states. (d) Energy diagram of exciplex showing the exchange splitting $\Delta_{\mathrm{ST}}$ between the singlet and triplet levels. For efficient TADF, $\Delta_{\mathrm{ST}}$ should be similar in magnitude to the thermal energy $k_{B} T$. The much quicker singlet recombination (with rate $k_{S}$ ) is depicted by the thicker arrow compared to triplet recombination (with rate $k_{T}$ ) and luminescence is assumed only for the singlet recombination. Spin mixing occurs due to the $\Delta g$ mechanism (i.e., transitions between $S$ and the activated $T_{0}$ level of $T^{*}$ ) and the hyperfine mechanism (i.e., transitions between $S$ and all activated triplet levels of $T^{*}$ ). (e) Theoretical calculations for the MEL $=\Delta \mathrm{EL} / \mathrm{EL}$ at large field with the $\Delta g$ mechanism $\left(B_{0 \mathrm{hf}}=0\right)$. (f) Theoretical calculations for the MEL $=\Delta \mathrm{EL} / \mathrm{EL}$ from hyperfine spin mixing at large field $(\Delta g=0)$. Increasing either $G_{T} / G_{S}$ or $B_{0 \mathrm{hf}}$ causes the MEL to be more negative for exciplex recombination values. Orange lines are $G_{T} / G_{S}=0.25$ and black lines are $G_{T} / G_{S}=1$. For panels (e) and (f), $k_{D}$ is the exciplex dissociation rate and $G_{S}$ is the singlet exciplex generation rate, whereas $G_{T}$ is the generation rate for thermally activated triplet exciplexes (see text for additional details). 
fields on excitons in organic semiconductors, and a theory is developed to describe exciplex magnetic-field effects. Magnetic-field effects in organic semiconductors occur when spin-carrying (paramagnetic) entities, such as electrons, holes, or triplet excitons, react with each other in spin-selective ways to form charge-neutral excitons [5], doubly charged bipolarons [16], trap-coupled carriers [17], or "trions" [18]. The spin-selective recombination can be influenced by local or global fields, or any other process that produces a magnetic-field-dependent reaction rate. When the reaction involves exciplexes, the electrons and holes are located on two distinct molecules, as shown in Fig. 1(a). In order to enhance the possibilities of exciplex formation, these two types of molecules are commonly evaporated together (coevaporated). For exciplexes the behavior of the electron and hole spins can differ considerably, as these electronic excitations reside on different molecules.

Spatially inhomogeneous (quasi)magnetic fields also lead to spin mixing $[10,11,14,15]$, including the $\Delta B$ mechanism, which arises in situations where there are large magnetic field gradients present, leading to a locally varying spin-precession frequency [10,11], and the $\Delta g$ mechanism [see Fig. 1(b)], which is very similar but the variation of the precession frequency is due to different proportionalities of the spin precession to the magnetic field (Landé $g$ factor) on neighboring molecules, as illustrated in Fig. 1(b). The $g$ factor differs from the free-electron value because of material-dependent interactions including spin-orbit coupling. The $\Delta g$ mechanism has recently been identified as the dominant MFE in several organic and hybrid perovskite systems [13-15].

Another major source of spin dynamics is the hyperfine mechanism [see Fig. 1(c)]. Every molecule, denoted by $i$, possesses a local hyperfine field, $\boldsymbol{B}_{\mathrm{hf}, i}$, on the order of $\mathrm{mT}$, due mainly to the magnetic moment of hydrogen nuclei about which the paramagnetic spin will precess. The hyperfine field direction varies randomly from site to site and therefore causes spin mixing in paramagnetic pairs on neighboring molecules. A spatially uniform externally applied field exceeding the hyperfine strength suppresses this spin mixing and therefore changes the reaction rate between the entities, which can have an amplified effect on transport in the percolative regime [19].

TADF exciplex devices are a promising material system for constructing organic semiconductor devices with immense magnetic field effects. In essence, a limit on the magnetic-field effect achievable via the so-called exciton mechanism is eliminated in TADF exciplex materials. In the exciton mechanism [5,7], electrons and holes located on neighboring molecules form a polaron pair, and spin mixing through the mechanisms above is permitted. The next step in the evolution of polaron pairs is the spinconserving formation of an exciton (in which both carriers inhabit the same molecule). Once the excitons form, because of the large $\Delta_{\mathrm{ST}}$, further spin mixing is not energetically allowed. Although the rates for singlet and triplet exciton formation rates are different, i.e., $k_{S} \neq k_{T}$, both channels are spin-allowed exothermic transitions. In contrast, for TADF exciplex materials in which $\Delta_{\mathrm{ST}}$ is of the order of the thermal energy, as shown in Fig. 1(d), singlet-triplet transitions readily occur upon thermal activation and exciplexes play the role of polaron pairs described in the exciton mechanism above. In these materials singlet exciplexes can decay radiatively directly to the singlet ground state, whereas triplet exciplexes cannot unless a spin flip occurs (i.e., phosphorescence). Therefore, the situation is that the singlet channel is spin allowed, whereas the triplet channel is spin "forbidden." Thus, $k_{S} \gg k_{T}$, and a very large MFE should be possible, for both the $\Delta g$ and hyperfine mechanisms.

The theoretical effects of $\Delta g\left(\equiv g_{1}-g_{2}\right)$ and hyperfine interactions on the spin dynamics and resulting MEL and magnetoconductance (MC) for TADF blends can be calculated from the stochastic Liouville equation for the two-spin density matrix $\rho$ [20]:

$$
\begin{aligned}
\frac{\partial \rho}{\partial t}= & -\frac{i}{\hbar}\left[H_{0}+H_{\Delta g}+H_{\mathrm{hf}}+H_{\mathrm{hf}, \Delta g}, \rho\right] \\
& -\frac{1}{2}\left\{k_{S} P_{S}+k_{T} P_{T}, \rho\right\}-k_{D} \rho+G,
\end{aligned}
$$

where the Hamiltonians are

$$
\begin{aligned}
H_{0} & =\frac{g_{1}+g_{2}}{2} \mu_{B} B \hat{z} \cdot\left(\boldsymbol{S}_{1}+\boldsymbol{S}_{2}\right), \\
H_{\Delta g} & =\frac{\Delta g}{2} \mu_{B} B \hat{z} \cdot\left(\boldsymbol{S}_{1}-\boldsymbol{S}_{2}\right), \\
H_{\mathrm{hf}} & =\frac{g_{1}+g_{2}}{2} \mu_{B}\left(\boldsymbol{B}_{\mathrm{hf}, 1} \cdot \boldsymbol{S}_{1}+\boldsymbol{B}_{\mathrm{hf}, 2} \cdot \boldsymbol{S}_{2}\right), \\
H_{\mathrm{hf}, \Delta g} & =\frac{\Delta g}{2} \mu_{B}\left(\boldsymbol{B}_{\mathrm{hf}, 1} \cdot \boldsymbol{S}_{1}-\boldsymbol{B}_{\mathrm{hf}, 2} \cdot \boldsymbol{S}_{2}\right),
\end{aligned}
$$

$P_{S}$ and $P_{T}=P_{T, 0}+P_{T,+}+P_{T,-}$ are the singlet and (total) triplet projection operators, and the curly braces denote the anticommutator. The two hyperfine fields are drawn from a Gaussian distribution with width $B_{0 \mathrm{hf}}$. $G$ is a diagonal matrix with elements $G_{S}$ and $G_{T,+}=G_{T, 0}=G_{T,-}=G_{T}$ for the generation of singlet and triplet exciplexes, respectively. We assume that all three triplet spin eigenstates have the same generation rate. We also assume that the rate of exciplex formation is unchanged by the presence of the magnetic field, and thus $G_{S}$ and $G_{T}$ are constant. Dissociation of the exciplex is also included in the theory with a rate $k_{D}$. We consider a steady-state condition and solve for the steady-state density matrix. EL is estimated from the singlet fraction of exciplexes as EL $=$ $k_{S} X_{S}=k_{S} \operatorname{Tr}\left[P_{S} \rho\right]$.

In general, solutions to Eq. (1) must proceed numerically. However, in the limit of negligible hyperfine fields, 
solutions for the $\Delta g$-induced MEL can be expressed analytically. In this case, we find

$$
\begin{aligned}
\mathrm{MEL}= & {\left[\frac{G_{T}\left(k_{D}+k_{S}\right)-G_{S}\left(k_{D}+k_{T}\right)}{G_{S}\left(2 k_{D}+k_{S}+k_{T}\right)}\right] } \\
& \times \frac{\left(\Delta g \mu_{B} B / \hbar\right)^{2}}{\left(k_{D}+k_{T}\right)\left(k_{D}+k_{S}\right)+\left(\Delta g \mu_{B} B / \hbar\right)^{2}} .
\end{aligned}
$$

The values of the MEL at large field for the $\Delta g$ mechanism, from Eq. (3), are shown in Fig. 1(e) for $k_{D}=0$ and for various values of $G_{T} / G_{S}$ and $k_{T} / k_{S}$. The situation for exciplex recombination corresponds to $k_{T} / k_{S} \ll 1$, which will allow magnetic field effects as large as $100 \%$ if $G_{T} / G_{S} \approx 1$, and produces a positive MEL. For exciton recombination $k_{T} / k_{S} \sim 1$, which produces a much smaller magnetic-field effect, as seen in Fig. 1(e). The sign of the MEL for the $\Delta g$ mechanism differs considerably from MEL caused by the hyperfine mechanism, for which a negative MEL is expected [21]. The values of the MEL at large field for the hyperfine interaction are shown in Fig. 1(f) for $G_{T} / G_{S}=1$ and $G_{T} / G_{S}=0.25$, and two values of the hyperfine field. A decrease in the $\Delta_{\mathrm{ST}}$ will produce a larger magnitude MEL in the experimentally relevant regime of $k_{T} / k_{S} \ll 1$, whether the mechanism is the $\Delta g$ mechanism or the hyperfine mechanism.

As the exciplex formation rate is directly related to the current through a device, the assumption of a constant formation rate corresponds to assuming a constant-current experimental condition for evaluating the effects of a magnetic field. Under conditions where the resistance changes substantially in the presence of a magnetic field, this assumption must be examined anew, and thus the MEL measured under constant-voltage conditions $\left(\left.\mathrm{MEL}\right|_{V}\right)$, for which the current and resistance change substantially, will differ from the MEL measured under constant-current conditions $\left(\left.\mathrm{MEL}\right|_{I}\right)$.

Our theory directly yields a magnetoluminescence response from the $\Delta g$ and hyperfine mechanisms, but, at first sight, does not address the magnetoconductance. As we present below, however, a sizable MC that is smaller but roughly comparable in magnitude (up to a factor of 2-3) is always measured alongside the MEL response. It was recognized in the early days of OMAR research that the exciton mechanism for MEL (which in this aspect behaves similarly to our exciplex mechanism for MEL) immediately produces a concomitant MC response [22]. In short, the electron and hole densities are large close to their respective injecting electrode. If the electron-hole recombination is efficient (and mutatis mutandis for inefficient recombination), there will be little spatial overlap of the two densities in the center of the device. This is because electrons and holes will immediately recombine in any part of the device where the two densities overlap. Therefore, the majority of the device is electrically charged, limiting the conductance by the so-called space-charge limited current law. Any magnetic-field-dependent recombination rate (as in our exciplex mechanism) will change the width of the portion of the device where the two densities overlap and spacecharge cancellation occurs, thus producing a change in the conductance. Thus, we assume the same functional dependence on magnetic field for the MC as for the MEL for this exciplex theory.

\section{DEVICE COMPOSITION AND FABRICATION}

Our TADF devices, schematically shown in Fig. 2(a), are thin-film devices with several layers deposited sequentially onto a glass slide with patterned indium-tin-oxide (ITO) electrodes. We use a materials combination for the primary layer that is known to produce a large internal electroluminescence quantum efficiency $\eta$ due to TADF [3]: 4,4,4-tris[3methylphenyl(phenyl)amino] triphenylamine (m-MTDATA) as donors and tris-[3-(3-pyridyl)mesityl]borane (3TPYMB) as acceptors. The m-MTDATA (>99\% pure) was purchased from Sigma-Aldrich and two batches with different purities of 3TPYMB ( $>99.2 \%$ pure, used for data in Figs. 9 and 10, and $>99.8 \%$ pure, used in all other figures) were purchased from Lumtec (Luminescence Technology Corp.). The materials were used as received.

After careful cleaning of the ITO-glass substrate in solvents, followed by plasma treatment, the organic layers are fabricated by thermal evaporation under high vacuum at $10^{-7}$ mbar. In our devices the primary layer consists of coevaporated m-MTDATA:3TPYMB with a mass fraction of $x \%$ 3TPYMB. All of our figures are for $x=75 \%$ unless otherwise stated, as this results in the largest magnetoelectroluminescence. A cathode layer consisting of calcium (covered by a protective layer of aluminum to increase air stability) is deposited by thermal evaporation in most devices, but $e$-beam evaporation is used for some devices. The active device area is about $0.6 \times 0.6 \mathrm{~mm}^{2}$.

The structure of the devices that give the largest effects is ITO/m-MTDATA $(15 \mathrm{~nm}) / 25$ wt \%-m-MTDATA: 3TPYMB $\quad(180 \mathrm{~nm}) / 3$ TPYMB $\quad(15 \mathrm{~nm}) / \mathrm{Ca} \quad(30 \mathrm{~nm}) / \mathrm{Al}$ (a)

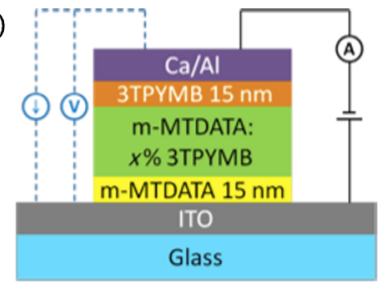

(b)

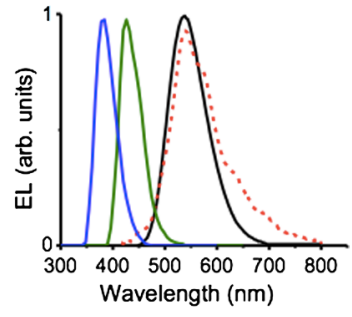

FIG. 2. Exciplex device structure and emission spectrum. (a) Schematic device structure showing the two measurement modes we use. The solid circuit is for a constant-voltage measurement, whereas the dashed circuit is for a constant current. (b) Electroluminescence spectrum for our device (red dashed line), compared with exciplex emission (solid black line) and exciton emission spectra (solid blue and green lines) taken from the literature [3]. This shows that exciplexes are responsible for EL in our devices. 
$(60 \mathrm{~nm})$. During the coevaporation of the $25 \mathrm{wt} \%-\mathrm{m}-$ MTDATA:3TPYMB, m-MTDATA is deposited at a rate of $0.1 \mathrm{~nm} / \mathrm{s}$ and 3 TPYMB at $0.3 \mathrm{~nm} / \mathrm{s}$. Figure $2(\mathrm{~b})$ shows the spectrum of the electroluminescence from these layers, compared with emission from excitons in either of the two constituents, and confirms that exciplexes indeed form in our devices [3].

The control devices, with organic luminescent or resistive layers of poly[2-methoxy-5-(2-ethylhexyloxy)1,4-phenylenevinylene] [MEH-PPV, see Fig. 3(a)] or tris (8-hydroxyquinolinato)aluminium $\left[\mathrm{Alq}_{3}\right.$, see Fig. 3(b)], consist of a thin film of the organic semiconductor sandwiched between a top and bottom electrode. The indium tin oxide $(100 \mathrm{~nm})$ coated glass substrates were obtained from Delta Technologies. The substrates are cleaned in an ultrasonic bath using acetone, methanol, and isopropanol followed by oxygen plasma cleaning. The conducting polymer poly(3,4-ethylenedioxythiophene)poly(styrenesulfonate) (PEDOT), purchased from Ossila Ltd., was spin coated at 4000 revolutions per minute (rpm) on top of the ITO to provide an efficient hole-injecting electrode. All other manufacturing steps were carried out in a nitrogen glovebox. The MEH-PPV (used as purchased from Sigma Aldrich) films were spin coated from a toluene solution with concentration $5 \mathrm{mg} / \mathrm{mL}$. The $\mathrm{Alq}_{3}$ (used as purchased from Sigma Aldrich) films were thermally evaporated in high vacuum onto the PEDOT-covered substrate. The organic semiconductor layer thickness was $\approx 150 \mathrm{~nm}$. The cathode layer consisting of calcium and aluminum was deposited by thermal evaporation or $e$-beam evaporation at a base pressure of $10^{-7}$ mbar on top (a)
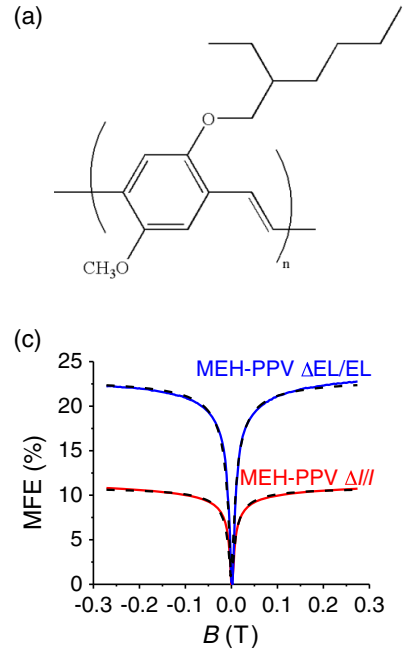

(b)

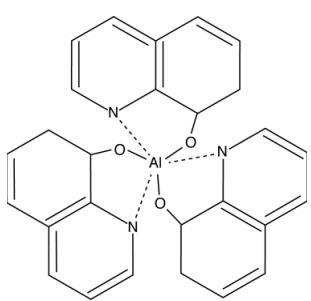

(d)

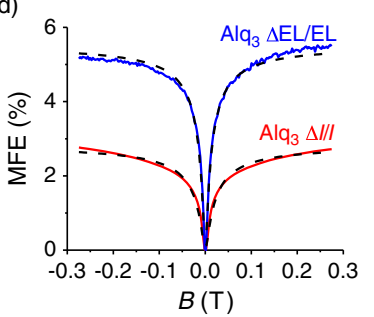

FIG. 3. Molecular structures of (a) MEH-PPV and (b) $\mathrm{Alq}_{3}$ that have been used in control experiments. Typical magnetconductance (red line) and magnetoelectroluminescence (blue line) in (c) MEH-PPV and (d) $\mathrm{Alq}_{3}$ control devices that are dominated by exciton emission. The dashed black lines represent fits to the non-Lorentzian line shape (see text). of the organic semiconductor layer. The active device area was roughly $0.6 \times 0.6 \mathrm{~mm}^{2}$.

MFE measurements are performed with the device mounted inside a closed-cycle He cryostat placed between two poles of an electromagnet. All data shown in this article are measured at room temperature, unless specified otherwise. The devices are driven at either a constant voltage $V$ or a constant current $I$ using a Keithley 2400 Source Meter. These particular current and voltage bias levels are chosen in pairs such that they corresponded to similar driving conditions. The electroluminescence intensity is measured using a photomultiplier tube that is shielded from the magnetic field during sweeps. For the optimal device conditioning procedure (see Sec. V), the devices are driven around a current density of $30 \mathrm{~mA} / \mathrm{cm}^{2}$ for $40 \mathrm{~min}$. Several MC or MEL traces are taken during the conditioning process to monitor the conditioning effectiveness and to find the optimal conditioning point.

The MFEs can be detected either by measuring $I$ as a function of $B$ or, alternatively, by measuring the EL as a function of $B$. These two measurements are performed simultaneously [see Fig. 2(a)] either when the applied device voltage is kept constant [measuring a change $\Delta I(B)$ and $\Delta \mathrm{EL}(B)]$ or when the device current is kept constant [measuring a change in applied voltage $\Delta V(B)$ and $\Delta \mathrm{EL}(B)]$. The MFE, $\Delta x / x$, in all these quantities is defined as $[x(B)-x(0)] / x(0)$.

Figure 3 shows the magnetic-field effects in MEH-PPV and $\mathrm{Alq}_{3}$ devices. These results serve as a reference point for the discussion of the new types of MFEs observed in the TADF devices. MEH-PPV and $\mathrm{Alq}_{3}$ are chosen because their MFEs have been extensively characterized in the OMAR literature [5,6] and device conditioning was first reported for MEH-PPV devices [23]. For typical MFEs the dependence of conductivity (or luminescence) on magnetic field is commonly either Lorentzian, $\Delta I(B) / I \propto$ $B^{2} /\left(B^{2}+B_{0}^{2}\right)$, or follows a specific non-Lorentzian form, $\Delta I(B) / I \propto B^{2} /\left(|B|+B_{0}\right)^{2}$, for the change in the current $I$, where $B_{0} \approx 5 \mathrm{mT}$ [6]. We find that the data in Fig. 3 can be accurately fitted by the non-Lorentzian expression.

\section{MAGNETIC-FIELD EFFECTS IN UNCONDITIONED EXCIPLEX DEVICES}

Figure 4 shows measurements of MFEs in as-prepared (unconditioned) devices for both constant-voltage and constant-current measurements. Figures 4(a) and 4(b) show that these devices exhibit sizable magnetoconductance and magnetoluminescence effects of up to $30 \%$ and $60 \%$, respectively, for constant-voltage measurements, whereas the effects for constant-current measurements are smaller. The data in Fig. 4 are well fit by the non-Lorentzian expression, as Fig. 3 is. The large (60\%) positive MEL is the largest reported in organic semiconductors and, remarkably, is completely at odds with the hyperfine 
Constant Voltage
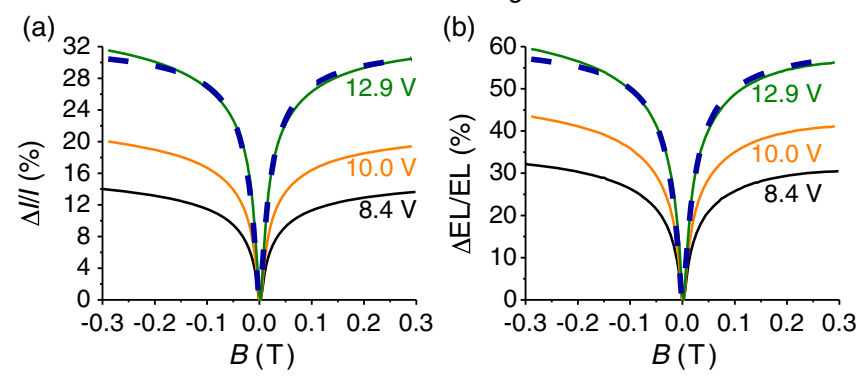

Constant Current
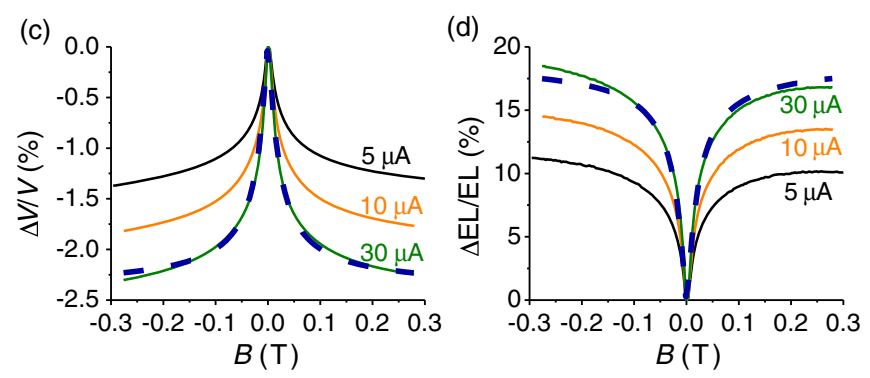

FIG. 4. (a) Magnetoconductance (MC) and (b) magnetoelectroluminescence (MEL) before device conditioning for three representative constant voltages. (c) Magnetovoltage and (d) magnetoelectroluminescence before device conditioning for three representative constant currents. The same color is used throughout the figure to designate measurements with a comparable current density. Thick dashed lines are non-Lorentzian (as defined in text) fits to the largest displayed curve in each panel.

mechanism where MEL $\sim-50 \%$ is predicted for exciplexes (where $k_{S}>k_{T}$ ).

However, our observations are consistent with the $\Delta g$ mechanism operating; the dominance of the $\Delta g$ mechansim is not surprising for MEL and MC in TADF materials because (1) the $g$ factors for two polaron spins $\left(\boldsymbol{S}_{1}\right.$ and $\left.\boldsymbol{S}_{2}\right)$ on different adjacent molecules $\left(g_{1}\right.$ and $\left.g_{2}\right)$ are expected to vary much more than for identical molecules and (2) McConnell's rule [24] states that $B_{0 \mathrm{hf}} \approx$ $(2-3 \mathrm{mT}) / \sqrt{N}$ in molecules with $N$ hydrogen atoms. Whereas our molecules in Fig. 1(a) do not contain many more hydrogens than other molecular organic semiconductors where hyperfine spin mixing is dominant, the rapid dissociation and association of the exciplex effectively further delocalizes the polarons and reduces the hyperfine interaction [25].

The data shown in Fig. 4 are fit to Eq. (3). During this fit $k_{S}$ and $\Delta g$ are kept constant. $k_{S}$ has been determined to be $3 \times 10^{6} \mathrm{~s}^{-1}$ for a similar combination of materials [3]. In other organic materials, $\Delta g$ has been found to be on the order of $10^{-3}-10^{-4}[26,27]$. We fix $\Delta g=10^{-4}$, corresponding to the low end of that spectrum. The crucial fitting parameters are $k_{D}$ and $G_{T} / G_{S} \cdot k_{D} \sim 10^{4} \mathrm{~s}^{-1}$, whereas $k_{T}$ is considerably smaller than $k_{D}$ and can therefore be assumed zero. Assuming that $G_{T}=G_{S}$ at high temperature, the value $G_{T} / G_{S}=0.19$ we find from our fit corresponds to an activation energy of $42 \mathrm{meV}$, consistent with the TADF behavior.

The maximum change in the electroluminescence with an applied field, relative to the zero-field electroluminescence, measured for a constant voltage $\left(\left.\mathrm{MEL}\right|_{V}\right)$ is 0.62 , whereas when measured for a constant current $\left(\left.\mathrm{MEL}\right|_{I}\right)$ it is 0.19 . The magnetic-field width of the curves at constant voltage and constant current are very similar, corresponding to $10.8 \mathrm{mT}$ at constant voltage and $10 \mathrm{mT}$ at constant current.

The observation that the MEL effect is considerably larger than the MC effect implies that an applied magnetic field leads to an enhancement in the device's electroluminescence efficiency. The electroluminescence power efficiency, defined as the light output power divided by the electrical input power, is proportional to $E L(B) / I(B)$ at constant voltage, and whereas the EL increases by up to $60 \%$, only $30 \%$ more current is drawn from the voltage source. Because both quantities, $\operatorname{EL}(B)$ and $I(B)$, are measured simultaneously in our experiments, we can readily plot the MFE on internal electroluminescence quantum efficiency $\eta$ versus $B$ (see Fig. 5). Our devices are designed for studies of the MFE and are not highperformance OLEDs; therefore, a comparison of the external quantum efficiency of our devices to those of highly optimized OLEDs reported in the literature would not be meaningful. The same material combination used here, however, has been utilized in highly efficient OLEDs [3].

TADF relies on a "reverse intersystem crossing" as its underlying mechanism, which in turn is just an umbrella term for spin-dependent singlet-triplet mixing interactions. We therefore uncover that the $\Delta g$ mechanism we study here makes a significant (and possibly dominant) contribution to this reverse intersystem crossing. Two interesting future research directions therefore become apparent: (i) the use of external $B$ fields to enhance the efficiency of TADF and

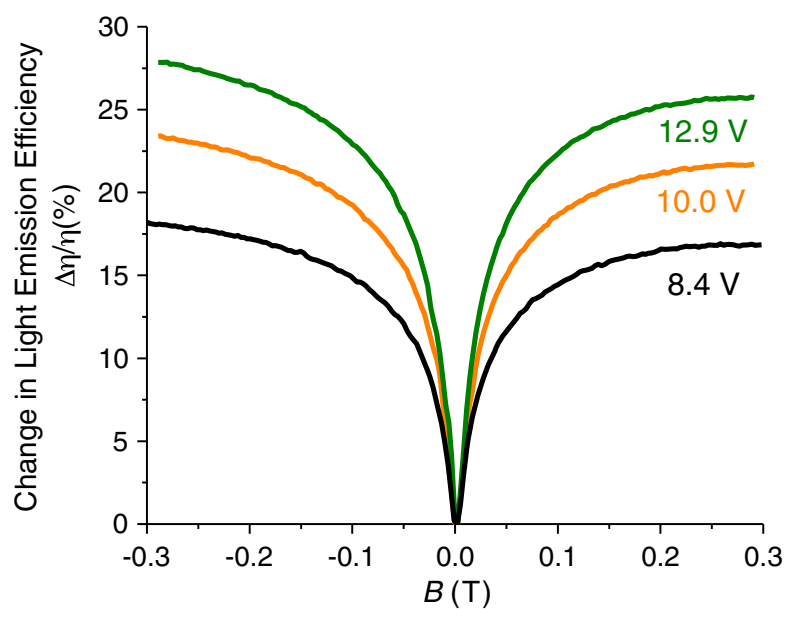

FIG. 5. Magnetic-field effect on electroluminescence efficiency $\eta$ in the pristine device for three representative constant voltages. The curves shown here are for the same device and experimental data set as in Figs. 4(a) and 4(b). 
ultimately exciplex OLEDs and (ii) the use of MFE spectroscopy to study the nature of the spin-dependent processes that are responsible for the reverse intersystem crossing.

\section{MFE ENHANCEMENT BY DEVICE CONDITIONING}

The effects seen in Fig. 4 are among the largest reported in the OMAR literature thus far, but Figs. 6(a) and 6(b) show that the effects, measured in the same device, increase spectacularly to over $500 \% \mathrm{MC}$ and $1000 \%$ MEL, after so-called "device conditioning" is performed. The effect of electrical conditioning of (excitonic) OMAR devices was reported by Niedermeier et al. [23], who found that the MC can be increased from $\approx 1 \%$ to $\approx 15 \%$ in MEH-PPV devices. Our conditioning procedure is similar to theirs, and consists of operating the device over a period of time at a relatively high current density. We note for later discussion that the increase in the constant-voltage MFE is much larger than the increase in the constant-current MFE, although even the constant-current MFE increase is over a factor of 2.

We now present a detailed description of the measurements made in the conditioned device that exhibits the largest MFE. These values are reproduced in several devices. Figure 7 shows MFE results as a function of various additional experimental parameters and device fabrication specifications, including temperature
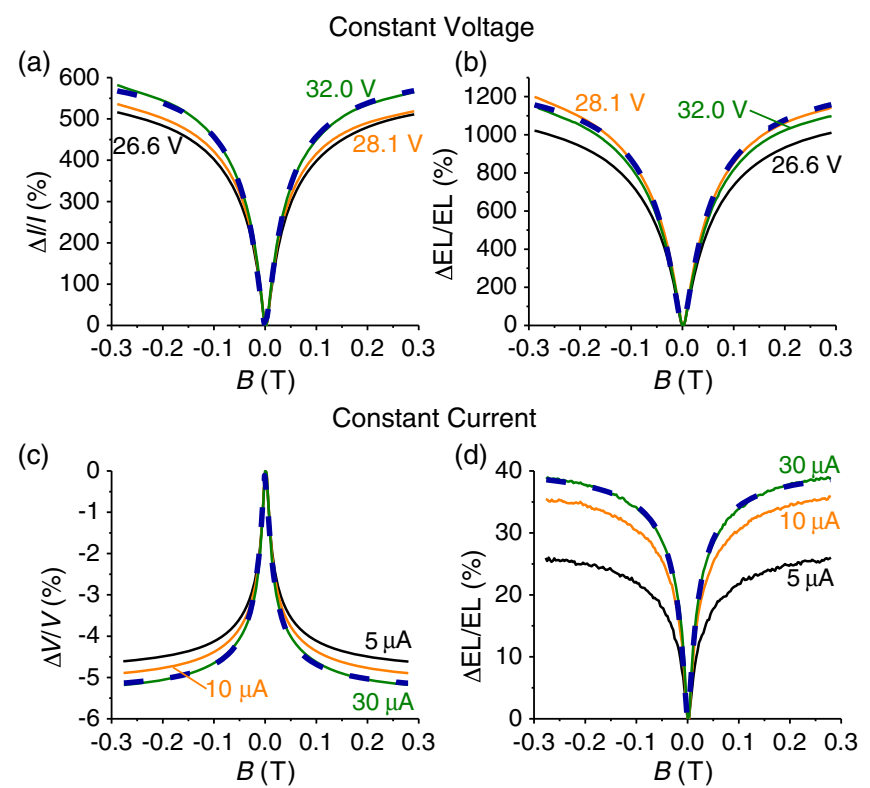

FIG. 6. (a) Magnetoconductance and (b) magnetoelectroluminescence after device conditioning for three representative constant voltages. (c) Magnetovoltage and (d) magnetoelectroluminescence after device conditioning for three representative constant currents. The same color is used throughout the figure to designate measurements with a comparable current density. Thick dashed lines are non-Lorentzian (as defined in text) fits to the largest displayed curve in each panel.
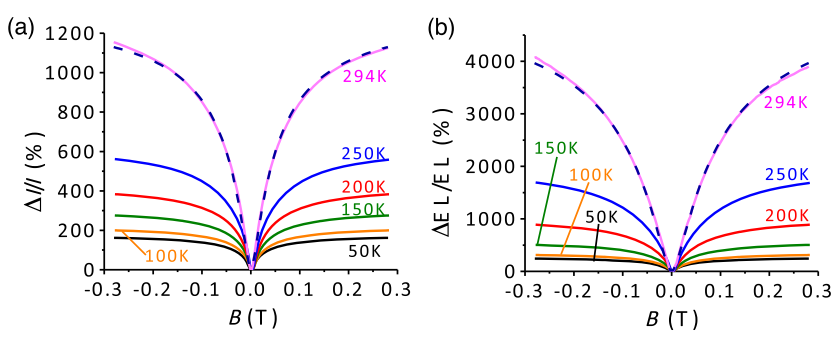

(c)
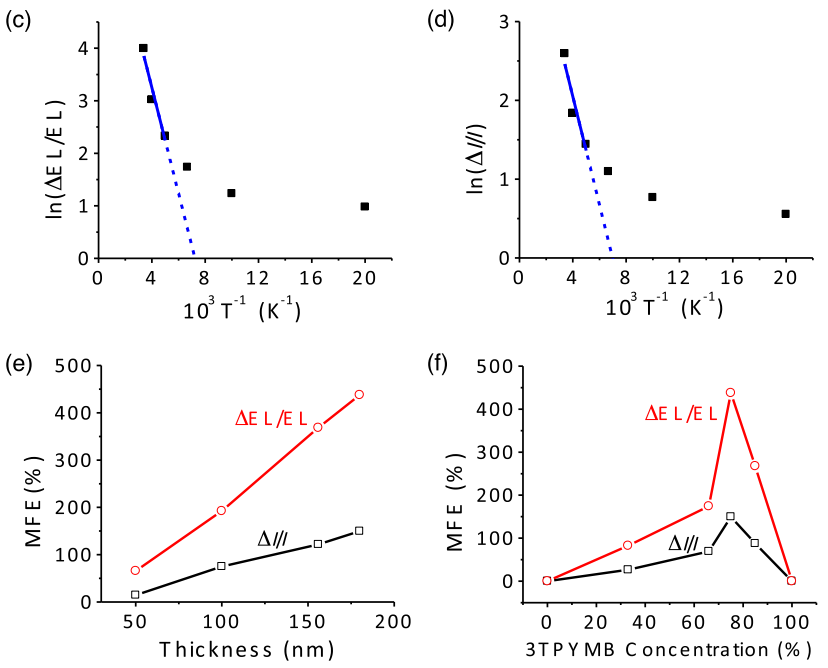

FIG. 7. (a),(b) Temperature dependence of magnetic-field effects in optimally conditioned device. Dashed lines are nonLorentzian (as defined in text) fits. (c),(d) Arrhenius plot of the saturation magnetic-field effect extracted from the data in (a) and (b), respectively. Solid lines are fits to an activated Boltzmann law indicating the range of data points used in the fit, whereas the dashed lines are extrapolations to lower temperatures. (e) Dependence of the saturation magnetoconductance $(\Delta I / I)$ and magnetoluminescence ( $\Delta \mathrm{EL} / \mathrm{EL}$ ) versus the thickness of the coevaporated layer, whereas (f) plots the same quantities versus the composition of the coevaporated layer.

[Figs. 7(a)-7(d)], thickness [Fig. 7(e)], and composition [Fig. 7(f)]. The devices shown in this figure follow the same design as the device used in the previous figure but are fabricated from a different batch of materials (see Sec. III). The effects we report are not due to local shorts or pinholes in the device, as even the optimally conditioned device we report here is stable and operates at a noise level equal to or better than that of the unconditioned device (see videos in Supplemental Material [28]). Figures 7(a) and 7(b) show that an even more dramatic increase in MC and MEL upon conditioning has been achieved in this device. $B_{0}$ increases to up to $\approx 50 \mathrm{mT}$ upon conditioning. This is much larger than the expected hyperfine strength, $B_{0 \mathrm{hf}}$, in our materials (also compare with Fig. 3).

Only those triplet exciplexes activated to near the singlet level [dashed lines, $T^{*}$, in Fig. 1(c)] can be involved in the conversion to singlets. Hence, $G_{T}<G_{S}$ in general, except at the highest temperatures when the two are equal. From this argument we expect the MFEs to increase with a rise in 
temperature (due to the increase in $G_{T}$ ), in agreement with experiment [Figs. 7(a) and 7(b)]. At very low temperatures the experimental data do not follow a simple Boltzmann law, but at temperatures above $200 \mathrm{~K}$, they agree with a Boltzmann dependence with an activation energy $\Delta_{\mathrm{ST}} \approx$ 60-80 meV [Figs. 7(c) and 7(d)]. This compares favorably with literature values [3] obtained from spectroscopic studies covering a similar temperature range as our Boltzmann fit. Figure 7(e) shows that the magnitude of the effect increases with increasing device thickness, and Fig. 7(f) shows that the effect is maximal for $x=0.75$ in the mixed layer. Adachi et al. found that $x=0.75$ leads to devices with maximum TADF efficiency [29].

\section{A. Relationship between constant-current MC and constant-voltage MC}

Figure 1(e) shows that the maximum predicted constantcurrent MEL is $100 \%$, which is consistent with our constant-current measurements, which do not surpass 40\% [see Fig. 6(d)]. Experimentally, the largest MFEs are observed in the constant-voltage mode, where the effects easily surpass $100 \%$. This is possible because the material combination in conjunction with the specified conditioning procedure yields highly nonlinear $I-V$ and $\mathrm{EL}-V$ curves.

Converting between the constant-voltage and constantcurrent modes of $\mathrm{MC}(\Delta I / I$ and $\Delta V / V$, respectively) can be carried out once the $I-V$ relationship is determined. We write the $I-V$ characteristics for magnetic field off and on as

$$
I_{0}=V^{\alpha}, \quad I_{B}=(V-\Delta V)^{\alpha},
$$

and by the definition of MC, we write

$$
\mathrm{MC}=\frac{I_{B}-I_{0}}{I_{0}}=\left(1-\frac{\Delta V}{V}\right)^{\alpha}-1
$$

which is an exact expression. By the binomial series expansion, we obtain leading order terms

$$
\mathrm{MC} \approx-\alpha \frac{\Delta V}{V}+\frac{\alpha}{2}(\alpha-1)\left(\frac{\Delta V}{V}\right)^{2}-\cdots
$$

The exponent of the nonlinearity $\alpha$ has been associated with trap-limited transport when the trap energies follow an exponential density of states [30]. Note that the MC at constant voltage increases linearly (to first order) with $\alpha$. Therefore, any process that increases $\alpha$ will likely increase the observed MC. This " $\alpha$ enhancement" allows the MFE at constant voltage to significantly exceed the $100 \%$ maximum predicted by our theory for the constantcurrent effect.

\section{B. Effect of electrical conditioning on $I V$ and $E L-V$ characteristics}

We now examine the effect of electrical conditioning on the current-voltage $(I-V)$ and electroluminescencevoltage $(E L-V)$ characteristics of the device. Figure 8 shows that $\alpha$ increases with device conditioning. Figure 8(a) shows the $I-V$ characteristics of the pristine device ("0 min") and the same device after several device conditioning operations (the current levels and conditioning times are assigned). Each conditioning step is performed on the device that was already conditioned by the cumulative prior steps. Data curves measured with an applied $B$ field of $0.3 \mathrm{~T}$ are shown as solid lines, and the zero-field characteristics are shown as dashed lines. The figure shows that the $I-V$ curves shift to higher voltages, with an onset voltage of about 5 and $25 \mathrm{~V}$ for the pristine and maximally conditioned device, respectively (further device conditioning would lead to a rapid device degradation and ultimately device failure). Both the $I-V$ and $\mathrm{EL}-V$ traces are approximately given by a power law with exponent $\alpha$. At the same time it is observed that the dependence of $I$ on $V$
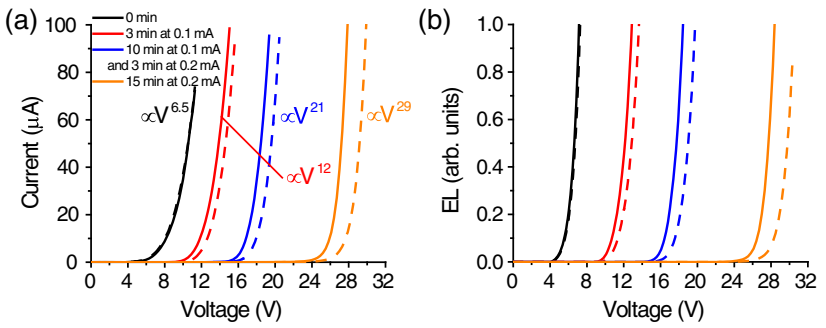

Constant Voltage
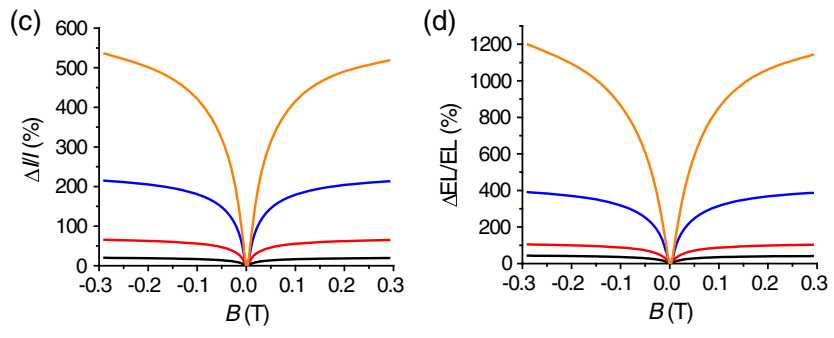

Constant Current
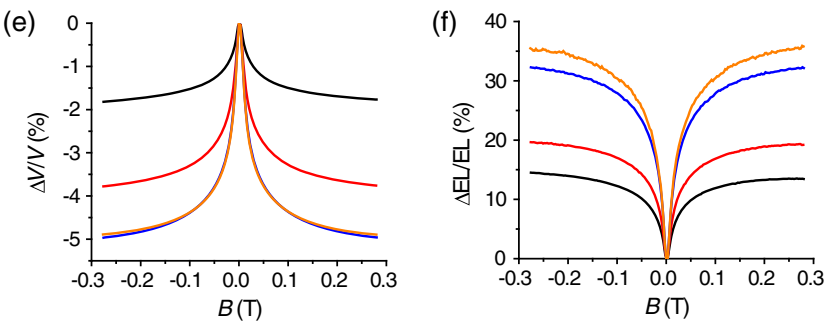

FIG. 8. (a) Current-voltage and (b) electroluminescencevoltage curves for different stages of device conditioning [protocols of the conditioning are specified as labels to the curves of (a); other panels use same color coding]. Dashed (solid) lines are measurements at $B=0(0.3)$ T. (c),(d) Constant-voltage and (e),(f) constant-current magnetic-field effect measurements for varying device conditioning. 
becomes increasingly nonlinear $\left(I \propto V^{\alpha}\right)$ with the exponent $\alpha$ increasing from approximately 6.5 to 30 . Comparing the solid and dashed lines, we find that phenomenologically the effect of the applied magnetic field is a parallel shift of the $I-V$ traces by a negative amount $\Delta V$. Similar behavior characterizes the EL $-V$ traces shown in Fig. 8(b).

\section{Effects of device conditioning on magnetic-field effects}

Figures 8(c) and 8(d) show the effect of device conditioning on MC and MEL measured at a constant applied voltage at several different stages of device conditioning [using the same color coding as for Fig. 8(a)], and Figs. 8(e) and 8(f) show the corresponding data for constant current measurements. Figures 8(c) and 8(d) show that the MC magnitude dramatically increases from about $20 \%$ for the pristine device to about $500 \%$ for the maximally conditioned device, and the EL magnitude increases from about $50 \%$ to about $1200 \%$. Device conditioning has a significantly less dramatic effect on the data shown in Figs. 8(e) and 8(f), which are for measurements at a constant current.

Table I shows the fitting results for different amounts of device conditioning. We fit the constant-current measurements with Eq. (3) by holding $k_{S}$ and $\Delta g$ constant, to the same values used in Sec. IV for the unconditioned devices, and setting $k_{T}=0$. We fit all data sets to the nonLorentzian expression. We find that the non-Lorentzian line shape results in excellent fits to the measured data, and the resulting fitting parameters are listed in Table I.

Noteworthy aspects of the fitting results are as follows. (i) $\alpha$ increases significantly from 13 to 32 upon conditioning, and a corresponding increase of the MEL $\left.\right|_{V}$ measured at constant voltage over the MEL $\left.\right|_{I}$ measured at constant current is observed. (ii) The electroluminescence efficiency $\eta$ decreases to $4 \%$ of the pristine value upon device conditioning. (iii) The curve width for constant voltage $\left.B_{0}\right|_{V}$ increases upon conditioning, but the curve width for constant current $\left.B_{0}\right|_{I}$ does not. (iv) The exciplex dissociation rate $k_{D}$ appears to be largely insensitive to conditioning, whereas conditioning moves the triplet-to-singlet branching ratio to favor triplet formation more. In terms of our theory, this indicates a reduction of the activation energy $\Delta_{\mathrm{ST}}$. Because $\Delta_{\mathrm{ST}}$ is a sensitive function of the electron-hole wave function overlap, a change in this quantity is to be expected if device conditioning results in changes to film morphology, molecular packing, or electronhole localization along the current flow paths. The increase in resistance associated with conditioning implies that conducting sites or electron-hole recombination sites are farther apart, which also is consistent with the reduction in $\Delta_{\mathrm{ST}}$. We note that further increase by conditioning in the MEL at constant current [which is connected directly to the $\Delta_{\mathrm{ST}}$ in our theory and Eq. (3)] should be possible since the regime of $\Delta_{\mathrm{ST}} \ll k_{B} T$ has not yet been reached. The increase of $\left.B_{0}\right|_{V}$ while $\left.B_{0}\right|_{I}$ is unchanged in conditioning appears related to the increase of $\alpha$ with conditioning.

Although the reduction of the electroluminescence efficiency is not a problem for magnetosensor applications, it is undesirable if the device is to be used as a magnetosensitive light-emitting device. However, there is no known reason why this reduction in efficiency should be a necessary companion to large MFEs. A procedure for achieving larger MEL without reducing $\eta$ may be found in the future, once the microscopic mechanism of conditioning is better understood. For example, we speculate that conditioning decreases $\Delta_{\mathrm{ST}}$ by increasing the average separation of the electron donor molecule and the electron acceptor molecule, which simultaneously increases the resistance. Finding molecule pairs with smaller $\Delta_{\mathrm{ST}}$ but higher conductivity could lead to larger MELs without compromising $\eta$. The exciplex model here will produce the largest MFEs for a system with a $\Delta_{\mathrm{ST}} \ll k_{B} T$, which is not a regime we reach even with conditioning.

It was shown previously [31] that the exposure of the organic layer to $\mathrm{x}$-ray bremsstrahlung that is generated

TABLE I. Effect of various device conditioning procedures on a square device $0.6 \mathrm{~mm}$ on a side (see Sec. III for details). The row number of the table signifies increasing conditioning times and/or currents. Specifically "Before device conditioning" is for zero conditioning time, "First device conditioning" is for 3 min conditioning at $0.1 \mathrm{~mA}$, "Second device conditioning" refers to an additional conditioning of $10 \mathrm{~min}$ at $0.1 \mathrm{~mA}$ plus an additional $3 \mathrm{~min}$ at $0.2 \mathrm{~mA}$, whereas "After device conditioning" signifies an additional conditioning for $15 \mathrm{~min}$ at $0.2 \mathrm{~mA}$ resulting in the optimally conditioned device. The first four columns contain directly measured quantities: MEL $\left.\right|_{V}\left(\left.\mathrm{MEL}\right|_{I}\right)$ is the maximum MEL measured for a constant voltage (current), $\left.B_{0}\right|_{V}\left(\left.B_{0}\right|_{I}\right)$ is the half width at quarter maximum and is also the parameter entering into the non-Lorentzian empirical law. The next two columns show quantities that can be calculated from the measured data without reference to any theory: $\alpha$ is the exponent of the device's nonlinear $I-V$ characteristics (see Sec. VA) and $\eta$ is the relative electroluminescence efficiency ( $\eta \equiv 1$ for the pristine device). The remaining quantities are extracted from fits to our theory (see text).

\begin{tabular}{lrccccccccc}
\hline \hline Device conditioning & $\left.\mathrm{MEL}\right|_{V}$ & $\left.\mathrm{MEL}\right|_{I}$ & $\left.B_{0}\right|_{V}(\mathrm{mT})$ & $\left.B_{0}\right|_{I}(\mathrm{mT})$ & $\frac{\left.\mathrm{MEL}\right|_{V}}{\mathrm{MEL} I_{I}}$ & $\alpha$ & $\eta$ & $k_{D}\left(\mathrm{~s}^{-1}\right)$ & $\frac{G_{T}}{G_{S}}$ & $\Delta_{\mathrm{ST}}(\mathrm{meV})$ \\
\hline Before device conditioning & 0.62 & 0.19 & 10.8 & 10 & 3.27 & 13.4 & 1 & $1 \times 10^{4}$ & 0.19 & 42 \\
First device conditioning & 1.15 & 0.23 & 11.3 & 9.5 & 5.00 & 16.2 & 0.38 & $9 \times 10^{3}$ & 0.23 & 37 \\
Second device conditioning & 4.00 & 0.35 & 14.5 & 9.5 & 11.4 & 24.1 & 0.16 & $9.2 \times 10^{3}$ & 0.35 & 26 \\
After device conditioning & 13.19 & 0.41 & 26.7 & 9.8 & 32.2 & 32.0 & 0.04 & $9.6 \times 10^{3}$ & 0.42 & 22 \\
\hline \hline
\end{tabular}


Thermal Evaporation
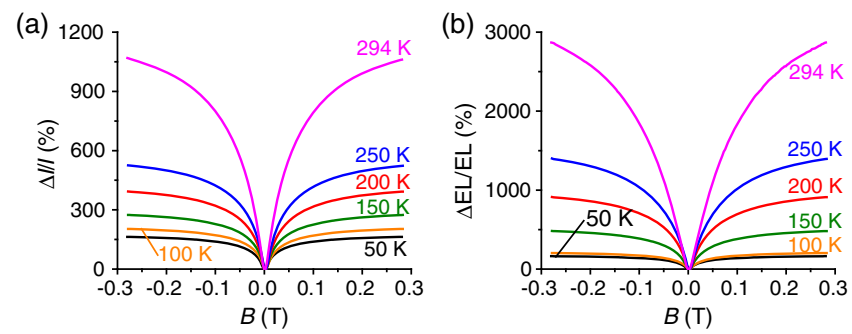

e-beam Evaporation
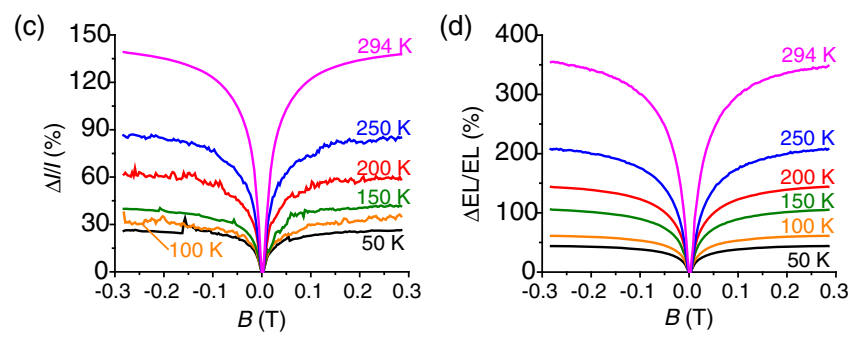

FIG. 9. Magnetoconductance and magnetoelectroluminescence in TADF-exciplex-based devices that differ in the fabrication procedure used to deposit the top metal electrode. (a),(b) Devices whose top electrode is fabricated by thermal evaporation. These devices have been maximally conditioned. (c),(d) Devices whose top electrode is fabricated by $e$-beam evaporation. These devices are not sensitive to device conditioning.

during the $e$-beam evaporation process can significantly increase the MFEs. It is therefore interesting to investigate whether devices fabricated using $e$-beam evaporation for the top electrode or cathode layer instead of thermal evaporation show a MFE enhancement similar to device conditioning. Figures 9(a) and 9(b) show MC and MEL measurements for the maximally conditioned thermally fabricated device as a function of temperature. We now compare these data for the thermally conditioned device to analogous data in an $e$-beam fabricated device [Figs. 9(c) and 9(d)]. Whereas the MFE in $e$-beam fabricated devices is large compared to unconditioned devices fabricated by thermal evaporation (see Sec. IV), it is a much smaller MFE than the thermally fabricated device after device conditioning (see Sec. V). In fact, we find that the $e$-beam devices are no longer sensitive to the device conditioning process. (a)

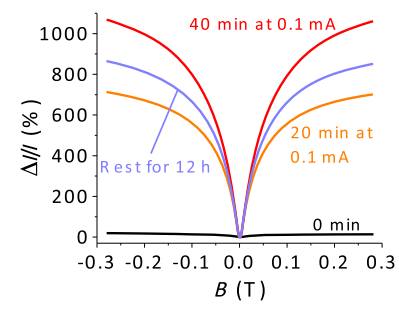

(b)

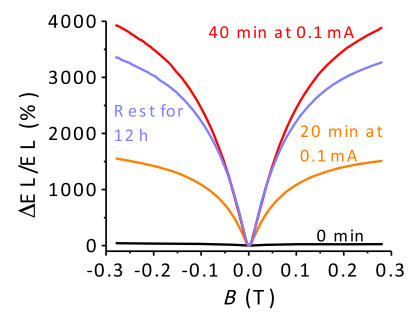

FIG. 10. (a),(b) Conditioning dependence of the device studied in Fig. 6. The blue line is for the optimally conditioned device after a rest period of $12 \mathrm{~h}$ and shows that the effect does not return to the pristine value.
For this reason, $e$-beam fabricated devices are the most suitable for studies of the dependence of MFEs on various parameters (such as layer thickness, layer composition, etc.) where an additional dependence on current density is not desired, as it would complicate the analysis. We therefore use $e$-beam devices for Figs. 7(e) and 7(f).

As a final comparison, we show the stability of the conditioning procedure. Figure 10 shows that the device does not return to its unconditioned value after resting for $12 \mathrm{~h}$. Further studies of the evolution of the magnetic field response with rest time will be the subject of future work.

\section{COMPARISON OF MAGNETIC FIELD EFFECTS IN EXCIPLEX DEVICES TO EXCITONIC DEVICES}

In this section, we compare MFE in our exciplex system to the (previously known) excitonic MFE. The simple, single-layer excitonic devices we study are not optimized to be efficient OLEDs, but serve as a reference for our MFE studies. Figures 11(a)-11(d) show that the magnitude of MFE in MEH-PPV also increases with electrical conditioning, but that the MFEs before and after conditioning are both much smaller than in the exciplex devices. The MFE of the $\mathrm{Alq}_{3}$ device is not sensitive to the electrical device conditioning, but it has previously been shown that a kind of device conditioning can be achieved by $\mathrm{x}$-ray exposure [31]. The value of $\Delta V / V$ and $\Delta E L / E L$ in MEH-PPV and $\mathrm{Alq}_{3}$ devices at constant current is less than one-third compared to the TADF exciplex devices. However, the value of $\Delta I / I$ and $\Delta \mathrm{EL} / \mathrm{EL}$ at constant voltage is smaller
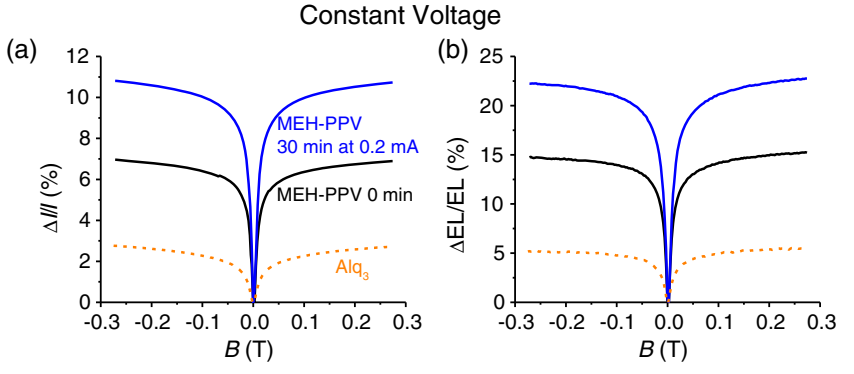

Constant Current
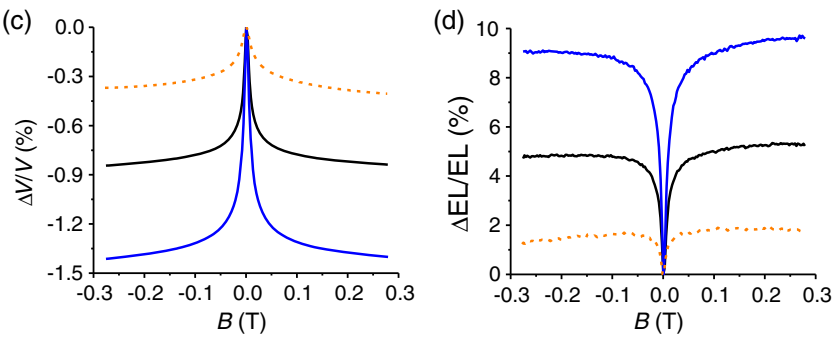

FIG. 11. Magnetotransport and magnetoluminescence in MEH-PPV and $\mathrm{Alq}_{3}$ devices. Magnetotransport and magnetoluminescence at (a),(b) constant voltage and (c),(d) constant current before and after device conditioning in MEH-PPV and $\mathrm{Alq}_{3}$ devices. 
TABLE II. Experimental values for $\Delta V / V$ and $\mathrm{MC}$ at different currents in MEH-PPV, $\mathrm{Alq}_{3}$, and m-MTDATA:3TPYMB devices. $\alpha$ is obtained from fitting the $I-V$ curves and the expected $\mathrm{MC}$ is calculated from Eq. (5).

\begin{tabular}{|c|c|c|c|c|}
\hline Description & $\alpha$ & $\Delta V / V$ & expected MC & $\mathrm{MC}$ \\
\hline \multicolumn{5}{|c|}{ MEH-PPV before device conditioning } \\
\hline $5 \mu A$ & 7.5 & -0.0088 & 0.068 & 0.074 \\
\hline $10 \mu A$ & 7.5 & -0.0085 & 0.065 & 0.07 \\
\hline $20 \mu A$ & 7.5 & -0.0081 & 0.063 & 0.068 \\
\hline \multicolumn{5}{|c|}{ MEH-PPV after device conditioning } \\
\hline $1 \mu A$ & 8.1 & -0.0166 & 0.142 & 0.131 \\
\hline $10 \mu A$ & 8.1 & -0.0143 & 0.122 & 0.109 \\
\hline $100 \mu A$ & 8.1 & -0.0085 & 0.071 & 0.071 \\
\hline \multicolumn{5}{|c|}{$\mathrm{Alq}_{3}$} \\
\hline $1 \mu A$ & 7.2 & -0.0056 & 0.041 & 0.041 \\
\hline $10 \mu A$ & 7.2 & -0.0041 & 0.03 & 0.028 \\
\hline $100 \mu A$ & 7.2 & -0.0033 & 0.024 & 0.024 \\
\hline \multicolumn{5}{|c|}{ m-MTDATA:3TPYMB at $30 \mu \mathrm{A}$} \\
\hline Before device conditioning & 13.43 & -0.024 & 0.372 & 0.325 \\
\hline First device conditioning & 16.24 & -0.039 & 0.857 & 0.695 \\
\hline Second device conditioning & 24.12 & -0.052 & 2.425 & 2.094 \\
\hline After device conditioning & 32 & -0.054 & 4.418 & 6.436 \\
\hline
\end{tabular}

than $2 \%$, which is much smaller than TADF devices. Moreover, the half width at quarter maximum of the traces $B_{0}$ in MEH-PPV and $\mathrm{Alq}_{3}$ devices is less than $5 \mathrm{mT}$, but for TADF exciplex devices, $B_{0}$ can be nearly $50 \mathrm{mT}$. We note that the reported data are first measured at a constant current from which we obtain $\Delta V / V$ and $\Delta \mathrm{EL} / \mathrm{EL}$ at constant current. Then we choose a voltage for the constant-voltage measurement that results in a similar current flow as for the constant-current measurements. In this way, we can perform an accurate, quantitative comparison between constant-current and constant-voltage measurements. See Table II for a summary of our results, where the observed value for the exponent $\alpha$, the measured magnetovoltage, the expected magnetoconductance due to the $\alpha$-enhancement, and finally the actually measured magnetoconductance are shown. The table shows good agreement between expected and actual magnetoconductance, validating our picture.

\section{CONCLUSION}

We show that TADF-based organic diodes can exhibit immense sensitivity to magnetic fields. The measured MFEs are the highest among systems with nonmagnetic components. Previous MFEs in organic semiconductors and colloidal quantum dots fail to surpass $20 \%[6,32]$. Similar MFE values are found in chemical reaction yields [33]. However, in hybrid perovskites, where $\Delta g$ is responsible for the MFEs, the MFEs are very small, $<0.5 \%$ [13]. Spin dynamics and the associated MFEs have also been recently studied for defects between either two nonmagnetic leads or one nonmagnetic lead and one magnetic lead. In both cases, the MFEs are less than $0.1 \%$ and appear to be due to hyperfine interactions $[34,35]$. In contrast to systems with magnetic components, in which the magnetic easy axes cause sensitivity to the vector character of the external magnetic field, the MFEs of these nonmagnetic TADFbased materials can be expected to depend predominately on the scalar magnitude of the field. Even for devices with magnetic components-for instance, those exhibiting tunneling magnetoresistance with maximum room temperature observed values $>600 \%$ [36] - our MFEs compare favorably despite other drawbacks inherent in the electronic properties of organic semiconductors when compared to metallic systems.

Our results imply that magnetic-field effects are a sensitive tool for investigating the spin-dependent exciplex physics that lies at the heart of TADF, and also shows that external and internal magnetic fields may serve as a booster of the electroluminescence efficiency in TADF devices. Our simple conditioning approach proves that immense MFEs are achievable in organic semiconductors, even though the microscopic processes that occur upon device conditioning cannot be identified with certainty. We speculate that conditioning introduces chemical changes to the molecules and/or changes to the film's nanoscale morphology that move the electron donors and acceptors effectively farther apart, both decreasing $\Delta_{\mathrm{ST}}$ and increasing the resistance. It remains a challenge to the organic semiconductor field to develop the necessary nanoscale tools to achieve smaller $\Delta_{\mathrm{ST}}$ 's while retaining higher conductivity, through morphological control during deposition or improved molecular design and targeted chemical synthesis.

\section{ACKNOWLEDGMENTS}

We acknowledge advice from J. Shinar on the coevaporation process. This work was supported in part by The University of Iowa Office of the Vice President for Research and Economic Development through the GAP and IFI Funding Programs. This material is based in part on work supported by the U.S. Department of Energy, Office of Science, Office of Basic Energy Sciences, under Award No. DE-SC0014336.

Y. W. and K. S.-T. contributed equally to this work.

[1] R. H. Friend, R. W. Gymer, A. B. Holmes, J. H. Burroughes, R. N. Marks, C. Taliani, D. D. C. Bradley, D. A. Dos Santos, J. L. Bredas, M. Logdlund, and W. R. Salaneck, Electroluminescence in Conjugated Polymers, Nature (London) 397, 121 (1999).

[2] L. J. Rothberg and A. J. Lovinger, Status of and Prospects for Organic Electroluminescence, J. Mater. Res. 11, 3174 (1996).

[3] K. Goushi, K. Yoshida, K. Sato, and C. Adachi, Organic Light-Emitting Diodes Employing Efficient Reverse Intersystem Crossing for Triplet-to-Singlet State Conversion, Nat. Photonics 6, 253 (2012). 
[4] H. Uoyama, K. Goushi, K. Shizu, H. Nomura, and C. Adachi, Highly Efficient Organic Light-Emitting Diodes from Delayed Fluorescence, Nature (London) 492, 234 (2012).

[5] J. Kalinowski, M. Cocchi, D. Virgili, P. Di Marco, and V. Fattori, Magnetic Field Effects on Emission and Current in $\mathrm{Alq}_{3}$-Based Electroluminescent Diodes, Chem. Phys. Lett. 380, 710 (2003).

[6] O. Mermer, G. Veeraraghavan, T. L. Francis, Y. Sheng, D. T. Nguyen, M. Wohlgenannt, A. Kohler, M. K. Al-Suti, and M.S. Khan, Large Magnetoresistance in Nonmagnetic $\pi$-Conjugated Semiconductor Thin Film Devices, Phys. Rev. B 72, 205202 (2005).

[7] V. N. Prigodin, J. D. Bergeson, D. M. Lincoln, and A. J. Epstein, Anomalous Room Temperature Magnetoresistance in Organic Semiconductors, Synth. Met. 156, 757 (2006).

[8] P. Desai, P. Shakya, T. Kreouzis, and W. P. Gillin, Magnetoresistance in Organic Light-Emitting Diode Structures under Illumination, Phys. Rev. B 76, 235202 (2007).

[9] B. $\mathrm{Hu}$ and $\mathrm{Y}$. Wu, Tuning Magnetoresistance between Positive and Negative Values in Organic Semiconductors, Nat. Mater. 6, 985 (2007).

[10] F. Wang, F. Macià, M. Wohlgenannt, A. D. Kent, and M. E. Flatté, Magnetic Fringe-Field Control of Electronic Transport in an Organic Film, Phys. Rev. X 2, 021013 (2012).

[11] F. Maciá, F. Wang, N. J. Harmon, M. Wohlgenannt, A. D. Kent, and M. E. Flatté, Organic Magnetoelectroluminescence for Room Temperature Transduction between Magnetic and Optical Information, Nat. Commun. 5, 3069 (2014).

[12] S. A. Crooker, F. Liu, M. R. Kelley, N. J. D. Martinez, W. Nie, A. Mohite, I. H. Nayyar, S. Tretiak, D. L. Smith, and P.P. Ruden, Spectrally Resolved Hyperfine Interactions between Polaron and Nuclear Spins in Organic Light Emitting Diodes: Magneto-electroluminescence Studies, Appl. Phys. Lett. 105, 153304 (2014).

[13] C. Zhang, D. Sun, C.-X. Sheng, Y. X. Zhai, K. Mielczarek, A. Zakhidov, and Z. V. Vardeny, Magnetic Field Effects in Hybrid Perovskite Devices, Nat. Phys. 11, 427 (2015).

[14] F. J. Wang, H. Bässler, and Z. V. Vardeny, Magnetic Field Effects in $\pi$-Conjugated Polymer-Fullerene Blends: Evidence for Multiple Components, Phys. Rev. Lett. 101, 236805 (2008).

[15] A. H. Devir-Wolfman, B. Khachatryan, B. R. Gautam, L. Tzabary, A. Keren, N. Tessler, Z. V. Vardeny, and E. Ehrenfreund, Short-Lived Charge-Transfer Excitons in Organic Photovoltaic Cells Studied by High-Field Magneto-Photocurrent, Nat. Commun. 5, 4529 (2014).

[16] P. A. Bobbert, T. D. Nguyen, F. W. A. van Oost, B. Koopmans, and M. Wohlgenannt, Bipolaron Mechanism for Organic Magnetoresistance, Phys. Rev. Lett. 99, 216801 (2007).

[17] N. J. Harmon and M. E. Flatté, Organic Magnetoresistance from Deep Traps, J. Appl. Phys. 116, 043707 (2014).

[18] P. Janssen, M. Cox, S. H. W. Wouters, M. Kemerink, M. M. Wienk, and B. Koopmans, Tuning Organic Magnetoresistance in Polymer-Fullerene Blends by Controlling Spin Reaction Pathways, Nat. Commun. 4, 2286 (2013).

[19] N. J. Harmon and M. E. Flatté, Spin-Flip Induced Magnetoresistance in Positionally Disordered Organic Solids, Phys. Rev. Lett. 108, 186602 (2012).
[20] R. Haberkorn, Density Matrix Description of Spin-Selective Radical Pair Reactions, Mol. Phys. 32, 1491 (1976).

[21] S. P. Kersten, A. J. Schellekens, B. Koopmans, and P. A. Bobbert, Magnetic-Field Dependence of the Electroluminescence of Organic Light-Emitting Diodes: A Competition between Exciton Formation and Spin Mixing, Phys. Rev. Lett. 106, 197402 (2011).

[22] J. D. Bergeson, V. N. Prigodin, D. M. Lincoln, and A. J. Epstein, Inversion of Magnetoresistance in Organic Semiconductors, Phys. Rev. Lett. 100, 067201 (2008).

[23] U. Niedermeier, M. Vieth, R. Patzold, W. Sarfert, and H. von Seggern, Enhancement of Organic Magnetoresistance by Electrical Conditioning, Appl. Phys. Lett. 92, 193309 (2008).

[24] H. M. McConnell and D. B. Chesnut, Theory of Isotropic Hyperfine Interactions in $\pi$-Electron Radicals, J. Chem. Phys. 28, 107 (1958).

[25] D. V. Dodin, A. I. Ivanov, and A. I. Burshtein, Hyperfine Interaction Mechanism of Magnetic Field Effects in Sequential Flourophore and Exciplex Flourescence, J. Chem. Phys. 138, 124102 (2013).

[26] C. F. O. Graeff, G. B. Silva, F. Nesch, and L. Zuppiroli, Transport and Recombination in Organic Light-Emitting Diodes Studied by Electrically Detected Magnetic Resonance, Eur. Phys. J. E 18, 21 (2005).

[27] Z. G. Yu, Spin-Orbit Coupling and Its Effects in Organic Solids, Phys. Rev. B 85, 115201 (2012).

[28] See Supplemental Material at http://link.aps.org/ supplemental/10.1103/PhysRevX.6.011011 for videos of the change in electroluminescence of the devices with magnetic field.

[29] C. Adachi, K. Goushi, and K. Yoshida, Delayed Fluorescence Material and Organic Electroluminescence Device, U.S. Patent No. 13/220,319 (30 August 2012), http://www.google.com/patents/US20120217869.

[30] P. Mark and W. Helfrich, Space-Charge-Limited Currents in Organic Crystals, J. Appl. Phys. 33, 205 (1962).

[31] J. Rybicki, R. Lin, F. Wang, M. Wohlgenannt, C. He, T. Sanders, and Y. Suzuki, Tuning the Performance of Organic Spintronic Devices Using X-Ray Generated Traps, Phys. Rev. Lett. 109, 076603 (2012).

[32] P. Guyot-Sionnest, D. Yu, P.-H. Jiang, and W. Kang, Spin Blockade in the Conduction of Colloidal CdSe Nanocrystal Films, J. Chem. Phys. 127, 014702 (2007).

[33] C. R. Timmel, U. Till, B. Brocklehurst, K. A. McLaughlin, and P. J. Hore, Effects of Weak Magnetic Fields on Free Radical Recombination Yields, Mol. Phys. 95, 71 (1998).

[34] A. G. Swartz, S. Harashima, Y. Xie, D. Lu, B. Kim, C. Bell, Y. Hikita, and H. Y. Hwang, Spin-Dependent Transport across $\mathrm{Co} / \mathrm{LaAlO} 3 / \mathrm{SrTiO} 3$ Heterojunctions, Appl. Phys. Lett. 105, 032406 (2014).

[35] H. Inoue, A. G. Swartz, N. J. Harmon, T. Tachikawa, Y. Hikita, M. E. Flatté, and H. Y. Hwang, Origin of the Magnetoresistance in Oxide Tunnel Junctions Determined through Electric Polarization Control of the Interface, Phys. Rev. X 5, 041023 (2015).

[36] S. Ikeda, J. Hayakawa, Y. Ashizawa, Y. M. Lee, K. Miura, H. Hasegawa, M. Tsunoda, F. Matsukura, and H. Ohno, Tunnel Magnetoresistance of $604 \%$ at $300 \mathrm{~K}$ by Suppression of Ta Diffusion in $\mathrm{CoFeB} / \mathrm{MgO} / \mathrm{CoFeB}$ Pseudo-Spin-Valves Annealed at High Temperature, Appl. Phys. Lett. 93, 082508 (2008). 